\title{
Solar system genealogy revealed by extinct short-lived radionuclides in meteorites
}

\author{
M. Gounelle ${ }^{1}$ and G. Meynet ${ }^{2}$ \\ ${ }^{1}$ Laboratoire de Minéralogie et de Cosmochimie du Muséum, UMR 7202, Muséum National d'Histoire Naturelle \& CNRS, \\ 75005 Paris, France \\ e-mail: gounelle@mnh. fr \\ 2 Geneva Observatory, University of Geneva, Maillettes 51, 1290 Sauverny, Switzerland
}

Received 14 February 2012 / Accepted 6 June 2012

\section{ABSTRACT}

\begin{abstract}
Context. Little is known about the stellar environment and the genealogy of our solar system. Short-lived radionuclides (SLRs, mean lifetime $\tau$ shorter than $100 \mathrm{Myr}$ ) that were present in the solar protoplanetary disk $4.56 \mathrm{Gyr}$ ago could potentially provide insight into that key aspect of our history, were their origin understood.

Aims. Previous models failed to provide a reasonable explanation of the abundance of two key SLRs, ${ }^{26} \mathrm{Al}\left(\tau_{26}=1.1 \mathrm{Myr}\right)$ and ${ }^{60} \mathrm{Fe}\left(\tau_{60}=3.7 \mathrm{Myr}\right)$, at the birth of the solar system by requiring unlikely astrophysical conditions. Our aim is to propose a coherent and generic solution based on the most recent understanding of star-forming mechanisms.

Methods. Iron-60 in the nascent solar system is shown to have been produced by a diversity of supernovae belonging to a first generation of stars in a giant molecular cloud. Aluminum-26 is delivered into a dense collected shell by a single massive star wind belonging to a second star generation. The Sun formed in the collected shell as part of a third stellar generation. Aluminum-26 yields used in our calculation are based on new rotating stellar models in which ${ }^{26} \mathrm{Al}$ is present in stellar winds during the star main sequence rather than during the Wolf-Rayet phase alone. Our scenario eventually constrains the time sequence of the formation of the two stellar generations that just preceded the solar system formation, along with the number of stars born in these two generations.

Results. We propose a generic explanation for the past presence of SLRs in the nascent solar system, based on a collect-injection-andcollapse mechanism, occurring on a diversity of spatial/temporal scales. In that model, the presence of SLRs with a diversity of mean lifetimes in the solar protoplanetary disk is simply the fossilized record of sequential star formation within a hierarchical interstellar medium. We identify the genealogy of our solar system's three star generations earlier. In particular, we show that our Sun was born together with a few hundred stars in a dense collected shell situated at a distance of 5-10 pc from a parent massive star having a mass greater than about 30 solar masses and belonging to a cluster containing $\sim 1200$ stars.
\end{abstract}

Key words. planets and satellites: formation - meteorites, meteors, meteoroids - ISM: clouds - gamma-ray burst: general stars: rotation

\section{Introduction}

Short-lived radionuclides (SLRs) are radioactive elements with mean lifetimes under $100 \mathrm{Myr}$ that were incorporated into meteorites' primitive components such as calcium- and aluminumrich inclusions (CAIs) or chondrules during the earliest evolution phases of our solar system. Understanding their origin has long been a major goal of cosmochemistry (Russell et al. 2001), as it is essential for constraining the stellar environment of the Sun at its birth (Meyer \& Clayton 2000), as well as for establishing a chronology of the solar system's first million years (McKeegan \& Davis 2005).

The SLRs with the longest mean lifetimes $(~ \gtrsim 5 \mathrm{Myr}$, such as ${ }^{129} \mathrm{I}\left[\tau_{129}=23.5 \mathrm{Myr}\right]$ or $\left.{ }^{244} \mathrm{Pu}\left[\tau_{244}=115 \mathrm{Myr}\right]\right)$ have abundances compatible with that of the expected Galactic background owing to continuous star formation on kpc spatial scales and tens of Myr timescales (Huss et al. 2009). Those SLRs with shorter mean lifetimes $(\$ 5 \mathrm{Myr})$ appear to be in excess relative to that same background abundance. Among these, ${ }^{10} \mathrm{Be},{ }^{36} \mathrm{Cl}$, and ${ }^{41} \mathrm{Ca}$ can be made by solar energetic particle irradiation of the protoplanetary disk (Gounelle et al. 2006; Duprat \& Tatischeff 2007; Jacobsen et al. 2011). For ${ }^{10} \mathrm{Be}$ alone, both an interstellar origin (Desch et al. 2004) and a solar-wind implantation model have also been evoked (Bricker \& Caffee 2010). The origin of two key SLRs, ${ }^{26} \mathrm{Al}\left(\tau_{26}=1.1 \mathrm{Myr}\right)$ and ${ }^{60} \mathrm{Fe}\left(\tau_{60}=3.7 \mathrm{Myr}\right)$, remains elusive.

After decades of measurements within CAIs (the first solids to have formed in our protoplanetary disk), the solar system's initial ${ }^{26} \mathrm{Al} /{ }^{27} \mathrm{Al}$ ratio is well established at $5.3 \times 10^{-5}$ (MacPherson et al. 1995; Jacobsen et al. 2008), though its homogeneity is still subject to debate (Villeneuve et al. 2009; Liu et al. 2012; Gounelle \& Russell 2005). The situation is a bit more complicated for ${ }^{60} \mathrm{Fe}$ because its record in CAIs is hampered by secondary processes and by nickel nucleosynthetic anomalies (Birck \& Lugmair 1988; Quitté et al. 2007), whose origin is far from being understood (Steele et al. 2011). Nickel-60 excesses attributed to the decay of ${ }^{60} \mathrm{Fe}$ were found in chondrules (Tachibana \& Huss 2003; Telus et al. 2011), which are believed to have formed $\sim 1$ Myr after CAIs (Villeneuve et al. 2009). Based on these data, the presently accepted upper limit of ${ }^{60} \mathrm{Fe} /{ }^{56} \mathrm{Fe}$ : in the nascent solar system is $\sim 3 \times 10^{-7}$ (Dauphas et al. 2008; Gounelle \& Meibom 2010; Telus et al. 2011).

To calculate the ${ }^{60} \mathrm{Fe}$ and ${ }^{26} \mathrm{Al}$ concentrations at the onset of solar system formation, in addition to the measured ratios presented above, we rely on the protosolar abundances given by Lodders (2003). With ${ }^{56} \mathrm{Fe} /{ }^{1} \mathrm{H}=3.2 \times 10^{-5},{ }^{27} \mathrm{Al} /{ }^{1} \mathrm{H}=$ $3.5 \times 10^{-6}$ and a hydrogen mass fraction of 0.71 (Lodders 2003), 
we obtain the following concentrations in the nascent solar system for ${ }^{26} \mathrm{Al}$ and ${ }^{60} \mathrm{Fe}: C_{\odot}\left[{ }^{26} \mathrm{Al}\right]=3.3 \times 10^{-9} M_{\odot} / M_{\odot}=3.3 \mathrm{ppb}$ (parts per billion) and $C_{\odot}\left[{ }^{60} \mathrm{Fe}\right]=4.0 \times 10^{-10} M_{\odot} / M_{\odot}=0.4 \mathrm{ppb}$. The initial ${ }^{26} \mathrm{Al} /{ }^{60} \mathrm{Fe}$ mass ratio was thus equal to 8.2 . Such elevated concentrations of ${ }^{26} \mathrm{Al}$ and ${ }^{60} \mathrm{Fe}$ need to be explained, and are the subject or the present paper.

Though asymptotic giant branch (AGB) stars have been proposed as a possible source of SLRs (Wasserburg et al. 2006; Trigo-Rodríguez et al. 2009; Lugaro et al. 2012), massive stars $\left(M \geq 8 M_{\odot}\right)$ are the best candidates to account for the presence of ${ }^{26} \mathrm{Al}$ and ${ }^{60} \mathrm{Fe}$ in the nascent solar system. This is because massive stars at all stages of their evolution are present in starforming regions, unlike AGB stars (Kastner \& Myers 1994).

The most massive stars $\left(M \gtrsim 25 M_{\odot}\right)$ burn hydrogen for millions of years on the main sequence (MS) before they enter the short-lived Wolf-Rayet (WR) phase that precedes the supernova (SN) explosion. Massive stars lose their nucleosynthetic products to the interstellar medium (ISM) via strong winds (during the MS and WR phase) and during the SN explosion. Interestingly, while ${ }^{60} \mathrm{Fe}$ is released only during the SN explosion, ${ }^{26} \mathrm{Al}$ is released during the MS, the WR, and the SN phases (Limongi \& Chieffi 2006; Palacios et al. 2005).

In the classical SN model, first proposed by Cameron \& Truran (1977), just after the discovery of ${ }^{26} \mathrm{Al}$ (Lee et al. 1976), ${ }^{26} \mathrm{Al}$ and ${ }^{60} \mathrm{Fe}$ were delivered together by a single SN (Boss et al. 2010; Ouellette et al. 2009) into the nearby solar protoplanetary disk or prestellar core. The distance, $r$, at which an SN needs to be in order to inject an SLR at the solar abundance into a phase (prestellar core or protoplanetary disk) having a linear size $r_{0}$ reads as (Cameron et al. 1995; Gounelle \& Meibom 2008):

$r=\frac{r_{0}}{2} \sqrt{\frac{\eta_{\mathrm{SN}} Y_{\mathrm{SN}}}{M_{\mathrm{SLR}}} \mathrm{e}^{-\Delta / \tau}}$

where $\eta_{\mathrm{SN}}$ is the mixing efficiency of the SN ejecta with the receiving phase, $Y_{\mathrm{SN}}$ is the SN yield of the considered SLR, $M_{\mathrm{SLR}}$ is the solar system mass of the SLR, $\Delta$ the time elapsed between the release of the radioactive element by the source and its incorporation in the receiving phase, and $\tau$ the mean lifetime of the SLR under scrutiny.

Because the solar system abundance of ${ }^{26} \mathrm{Al}$ is far better constrained than that of ${ }^{60} \mathrm{Fe}$, we use the former SLR $\left(C_{\odot}\left[{ }^{26} \mathrm{Al}\right]=\right.$ $3.3 \mathrm{ppb})$ to calculate the maximum distance at which the $\mathrm{SN}$ has to be from either the disk or the core to deliver SLRs at the solar abundance. Using the SN yields of massive stars with $M \leq 60 M_{\odot}$ (Huss et al. 2009) calculated by Woosley \& Heger (2007), we obtain $r \leq 0.4$ pc for a disk of mass $0.013 M_{\odot}$ (Hayashi et al. 1985; Ouellette et al. 2005) and size $r_{0}=100 \mathrm{AU}$ with an injection efficiency of 0.7 (Ouellette et al. 2009), and $r \leq 0.6 \mathrm{pc}$ for a core of mass $2 M_{\odot}$ and size $r_{0}=0.058 \mathrm{pc}$ with an injection efficiency of 0.02 (Boss \& Keiser 2010). The receiving phases parameters (mass and size) correspond to the observed properties of disks and cores adopted by the tenants of the single SN model (Ouellette et al. 2005; Boss \& Keiser 2010), while the injection efficiencies are estimated by the same authors (Ouellette et al. 2009; Boss \& Keiser 2010). As we conservatively assumed $\Delta=0$, the calculated distances are upper limits of the maximum distances. In other words, if we applied a decay interval of $\Delta \gtrsim 1 \mathrm{Myr}$ as required by all SN models (Huss et al. 2009), it would imply that the receiving phases (disk or core) lie at a few tenths of a parsec from the SN at most to receive ${ }^{26} \mathrm{Al}$ at the solar abundance.

It is very unlikely, if not impossible, however, to find a protoplanetary disk or a dense core that close to an SN. Before they explode as $\mathrm{SNe}$, massive stars carve large ionized regions in the ISM (called HII regions) where the gas density is too low and temperature too high for star formation to take place (Bally 2008). Observations show that even around a massive star that still needs to evolve for $2 \mathrm{Myr}$ before it explodes as a SN, disks and cores are found several parsecs away (Hartmann 2005), too far to receive ${ }^{26} \mathrm{Al}$ and ${ }^{60} \mathrm{Fe}$ at the solar abundance. In addition, because $\mathrm{SNe}$ ejecta are vastly enriched in ${ }^{60} \mathrm{Fe}$ relative to ${ }^{26} \mathrm{Al}$ and their respective solar abundances (Woosley \& Weaver 2007), all models relying on $\mathrm{SN}$ injection lead to a ${ }^{26} \mathrm{Al} /{ }^{60} \mathrm{Fe}$ ratio that is far lower than the initial solar ratio of 8.2, unless special conditions are adopted (Desch et al. 2011).

Following the pioneering work of Arnould et al. (1997), WR stars winds have recently been reconsidered as a specific source for ${ }^{26} \mathrm{Al}$ alone (Arnould et al. 2006; Gaidos et al. 2009; Tatischeff et al. 2010). In the model of Gaidos et al. (2009), which considers injection of ${ }^{26} \mathrm{Al}$ at the molecular cloud scale, very specific conditions (such as a precise timing between the formation of massive stars and the Sun or stellar clusters with an extremely large number of stars) are needed. In the stimulating model of Tatischeff et al. (2010), ${ }^{26} \mathrm{Al}$ is delivered into a bowshock-produced shell by a single runaway massive star moving with a velocity $\geq 20 \mathrm{~km} \mathrm{~s}^{-1}$ in a dense $\left(n \sim 100 \mathrm{~cm}^{-3}\right)$ starforming region. At such a velocity ( $20 \mathrm{pc} / \mathrm{Myr})$, the runaway WR star considered by Tatischeff et al. (2010) would escape any dense star-forming region with size $\geq 40$ pc within $2 \mathrm{Myr}$, preventing the collection of dense gas well before the entry into the WR phase. In addition none of these models is generic (i.e. they fail to offer a common explanation for ${ }^{60} \mathrm{Fe}$ and ${ }^{26} \mathrm{Al}$ ), nor do they constrain the solar system's genealogy. Finally, because of the rarity of WR stars (Crowther 2007), such models require a special explanation for the formation of the solar system.

The goal of the present work is to identify a coherent model that accounts for the presence of ${ }^{26} \mathrm{Al}$ and ${ }^{60} \mathrm{Fe}$ in the nascent solar system and which is in line with the most recent astronomical observations of star-forming regions (Sect. 2). The proposed origin for ${ }^{26} \mathrm{Al}$ (Sects. 2 and 3 ) is entirely original and relies on new rotating models of massive stars. The proposed explanation for ${ }^{60} \mathrm{Fe}$ (Sect. 4) is an update of the Supernova Propagation \& Cloud Enrichment (SPACE) model elaborated by Gounelle et al. (2009). Combining these two results, a generic explanation is offered for the presence of SLRs having a diversity of mean lifetimes in the early solar system (Sect. 5). In Sect. 6, we discuss our results, focusing on the newly established solar system genealogy.

\section{Model sketch}

We propose to explain the SLR abundances in the nascent solar system within the framework of a common picture of star formation (Hennebelle et al. 2009), namely that of sequential star formation within a spatially and temporally structured giant molecular cloud (GMC). We consider the following sequence of events (see Fig. 1):

- Time 0 is that of the formation of a first generation (\#1) of $N_{1}$ stars in a region \#1 of the GMC (see panel $a$ of Fig. 1).

- After a few Myr, massive stars from generation \#1 explode as $\mathrm{SNe}$ and start to deliver ${ }^{60} \mathrm{Fe}$ into a neighboring region \#2.

- Five Myr after time 0 (see in Sect. 4.1 how this time is estimated), an ${ }^{60} \mathrm{Fe}$ steady-state abundance (due to the balance between decay and production by $\mathrm{SNe}$ from generation \#1) is established in region \#2. 

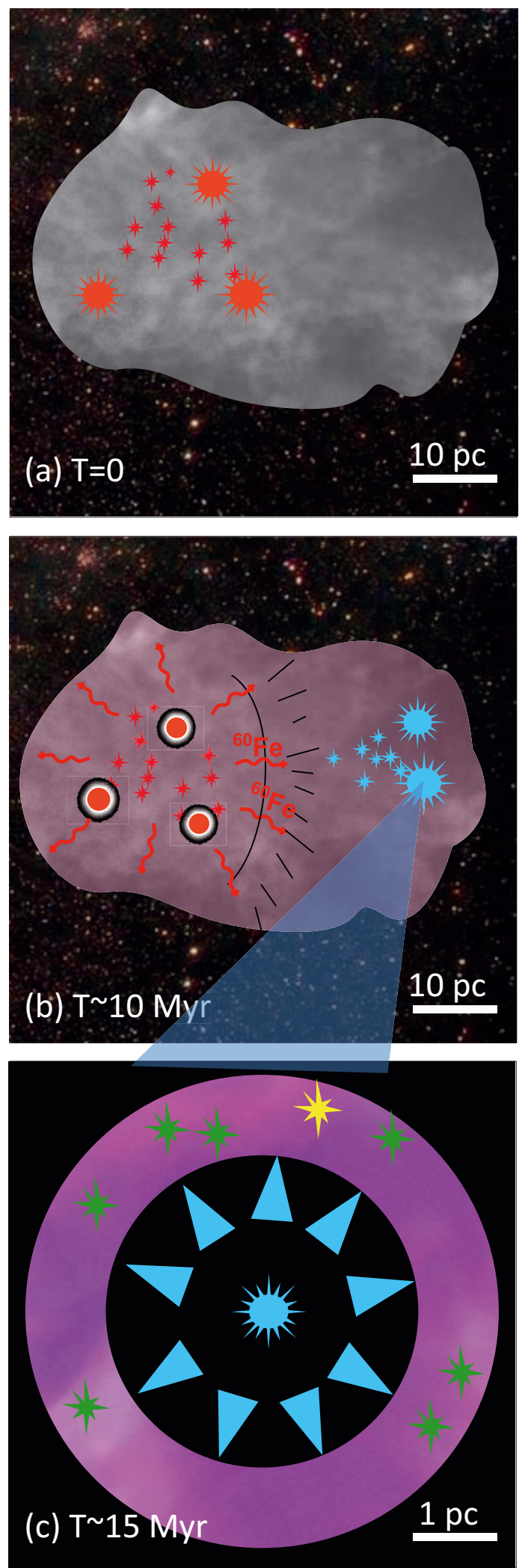

Fig. 1. Sketch of the model described in Sect. 2. Star generations \#1, $\# 2$, and \#3 are respectively in red, blue, and green. In panel b), $\mathrm{SNe}$ remnants are shown with a dark contour. The reddish background symbolizes ${ }^{60} \mathrm{Fe}$ delivered by the $\mathrm{SNe}$ belonging to the first star generation. In panel c), the purple color of the shell symbolizes the combination of ${ }^{26} \mathrm{Al}$ delivered by the single massive star wind from generation \#2 and the ${ }^{60} \mathrm{Fe}$ delivered by generation \#1 stars. Our Sun, born in the circumstellar shell together with a few hundreds fellow low-mass stars (generation \#3), is shown in yellow.
Main sequence $(0)$

WR SN

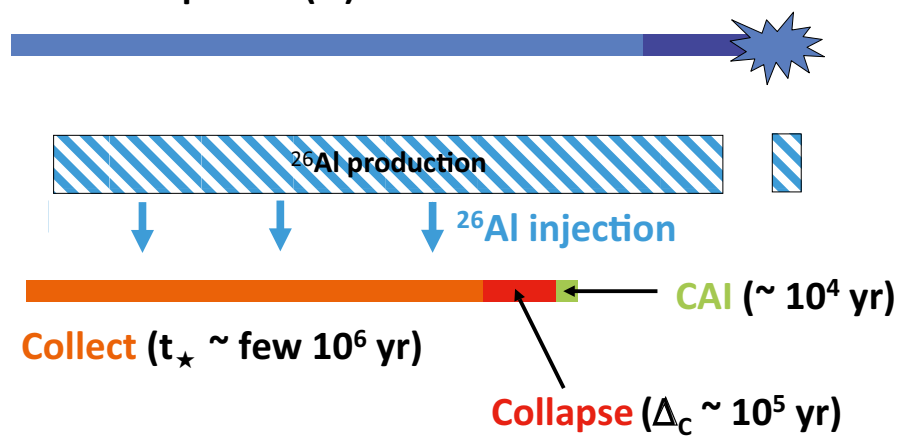

Fig. 2. Timeline for ${ }^{26} \mathrm{Al}$ injection in a dense collected shell around a massive star, starting approximately $10 \mathrm{Myr}$ after the birth of stellar generation $\# 1$ and lasting $\sim t_{\star}$. The upper part of the figure represents the time line of the central massive star of panel $c$ in Fig. 1. In the middle part, the timeline of the ${ }^{26} \mathrm{Al}$ production and injection (arrows) is shown. Aluminum-26 is present in the wind some $10^{5} \mathrm{yr}$ after the star formation and absent when products of helium-burning appear at the surface (WC-type star) since ${ }^{26} \mathrm{Al}$ is destroyed in helium-burning cores. It is present while the star explodes as an SN. Aluminum-26 injection in the shell lasts during the whole collection phase and is assumed to stop during the collapse phase lasting a time $\Delta_{C} \sim 10^{5} \mathrm{yr}$. The lower part represents the timeline of the collected shell, which eventually collapses to produce a protoSun and a protoplanetary disk in which most CAIs form.

- At $t \sim 10 \mathrm{Myr}$, a second star generation (\#2, containing a total of $N_{2}$ stars) forms in region \#2, partly due to the compressive action of the generation \#1 SNe shockwaves onto the molecular cloud gas (Preibisch \& Zinnecker 2007) (see panel $b$ of Fig. 1).

- From $t \sim 10 \mathrm{Myr}$ and for some Myr, the wind of one or two massive stars from generation \#2 will collect ISM gas to build a dense shell surrounding an HII region of radius 5-10 pc (Deharveng et al. 2010). That collected shell, which contains ${ }^{60} \mathrm{Fe}$ originating in the $\mathrm{SNe}$ of the first generation, will be wind-enriched in ${ }^{26} \mathrm{Al}$ via efficient turbulent mixing (Koyama \& Inutsuka 2002) during a time $t_{\star}$ lasting a few Myr (see Fig. 2).

- At $t \sim 10 \mathrm{Myr}+t_{\star}$, aluminum-26 delivery ends when the dense shell fragments and collapses via a diversity of gravito-turbulent mechanisms, such as gravitational instabilities and ionization of a turbulent medium (Deharveng et al. 2010), to form a third generation star cluster (\#3). The collapse phase lasts $\Delta_{C} \sim 10^{5}$ yr. Our Sun belongs to that third generation of stars (see panel $c$ of Fig. 1). CAIs formed in the protoplanetary disk surrounding the protoSun on a timescale of a few $10^{4} \mathrm{yr}$ (Jacobsen et al. 2008) contains ${ }^{26} \mathrm{Al}$ from the wind of the generation \#2 massive star and ${ }^{60} \mathrm{Fe}$ from the generation \#1 SNe (Fig. 2).

In the following, we show that such a scenario presents many realizations that permit a good fit of the quantities of ${ }^{26} \mathrm{Al}$ and ${ }^{60} \mathrm{Fe}$ at the solar system's birth. In that respect, it provides a more natural explanation than previous models, because it does not require special and/or unlikely conditions, and leads to understanding how the solar system forms within a broader perspective. The key point is that ${ }^{60} \mathrm{Fe}$ comes from the $\mathrm{SNe}$ of the first generation of stars, while ${ }^{26} \mathrm{Al}$ comes from the wind of a single massive star belonging to a second star generation. This decoupling between the sources of ${ }^{60} \mathrm{Fe}$ and of ${ }^{26} \mathrm{Al}$ is essential to explain the observed ${ }^{26} \mathrm{Al} /{ }^{60} \mathrm{Fe}$ ratio when the solar system 
formed, and it does appear as a signature of the sequential star formation process.

\section{Origin of ${ }^{26} \mathrm{Al}$ in the solar system}

\section{1. ${ }^{26}$ Al yields in massive stars winds}

The ${ }^{26} \mathrm{Al}$ yields during the wind phase are calculated using new models for massive stars (Fig. 3). These models present two major improvements over previous ones (Limongi \& Chieffi 2006; Palacios et al. 2005) considered so far by modelers (Arnould et al. 1997; Gaidos et al. 2009; Tatischeff et al. 2010). First, they are built on newly determined initial solar abundances (Asplund et al. 2009). The solar abundances determined by Asplund et al. (2009) are similar to those shown by present-day massive stars in the solar neighborhood, and this composition is also quite similar to those expected in massive stars at the formation of the solar system (see Table 5 in Asplund et al. 2009).

Second, these models account for the effects of axial rotation and improved mass loss rates (Meynet et al. 2008). Rotational mixing is accounted for as in Palacios et al. (2005). More precisely, rotational mixing accounts for the effects of meridional currents and shear turbulence. The vertical shear diffusion was taken as given by Talon \& Zahn (1997), and the horizontal shear diffusion was taken as in Maeder (2003). A moderate overshooting is included. The radii of the convective cores given by the Schwarzschild's criterion are increased by $0.1 H_{\mathrm{p}}$, where $H_{\mathrm{p}}$ is the pressure scale height estimated at the Schwarzschild boundary. The mass loss rates are those of Vink et al. $(2000,2001)$ and of de Jager et al. (1988) outside the application domain of the prescriptions by Vink et al. (2000, 2001). During the Wolf-Rayet phase, we use the mass loss rates of Nugis \& Lamers (2000). The impact of rotation on the mass loss rates are accounted for as in Maeder \& Meynet (2000).

These models reproduce reasonably well the observed number ratio in the solar vicinity of WR to O-type stars, of nitrogenrich (WN stars) to carbon-rich (WC) WR stars, and of the transition WN/C to WR stars (Meynet et al. 2008). Because these ratios are sensitive to both rotation and mass loss, these good agreements provide some support to the way these processes are included in the present stellar models.

We stress here that rotational mixing allows some products synthesized in the core to appear at an early stage of the evolution of massive stars. For instance, while in non-rotating models, one has to wait until the H-rich envelope is nearly completely removed by the stellar winds in order for the star to eject ${ }^{26} \mathrm{Al}$, in rotating models the surface is already ${ }^{26} \mathrm{Al}$-enriched during earlier phases thanks to diffusion of ${ }^{26} \mathrm{Al}$ from the core to the surface through the (shear unstable) radiative layers. This lengthens the period during which the wind is ${ }^{26} \mathrm{Al}$-enriched (Fig. 3). This period is no longer reduced to the WR phase as in non-rotating models, but also covers part of the MS phase.

\section{2. ${ }^{26} \mathrm{Al}$ abundance in the collected shell}

As already explained above, the wind of one or two massive stars from generation \#2 (containing a total of $N_{2}$ stars) will collect ISM gas to build a dense shell. The dependence with time of aluminum-26 concentration in the shell, $C\left[{ }^{26} \mathrm{Al}\right]$, reads as $C\left[{ }^{26} \mathrm{Al}\right]=\eta_{\text {wind }} P_{\text {wind }}\left[{ }^{26} \mathrm{Al}\right](t) / M_{\text {shell }}$, where $\eta_{\text {wind }}$ is the mixing efficiency of the wind with the shell, $M_{\text {shell }}$ is the mass of the collected shell and $P_{\text {wind }}\left[{ }^{26} \mathrm{Al}\right]$ is the quantity of non-decayed ${ }^{26} \mathrm{Al}$ (i.e. survivor) present in the total wind ejecta of the massive star at time $t$. Imposing that the Sun forms in that shell after

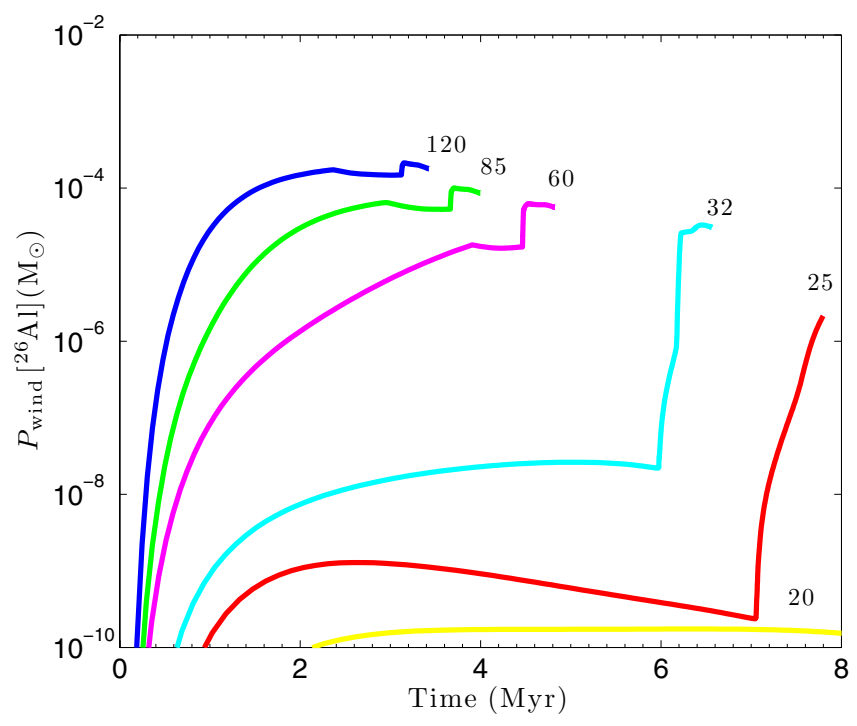

Fig. 3. Integrated ${ }^{26} \mathrm{Al}$ abundance produced by the stellar wind of massive stars as a function of time. Labels indicate stars' initial masses (in $M_{\odot}$ ). These numbers take ${ }^{26} \mathrm{Al}$ radioactive decay into account (see Eq. (1) of Arnould et al. 2006).

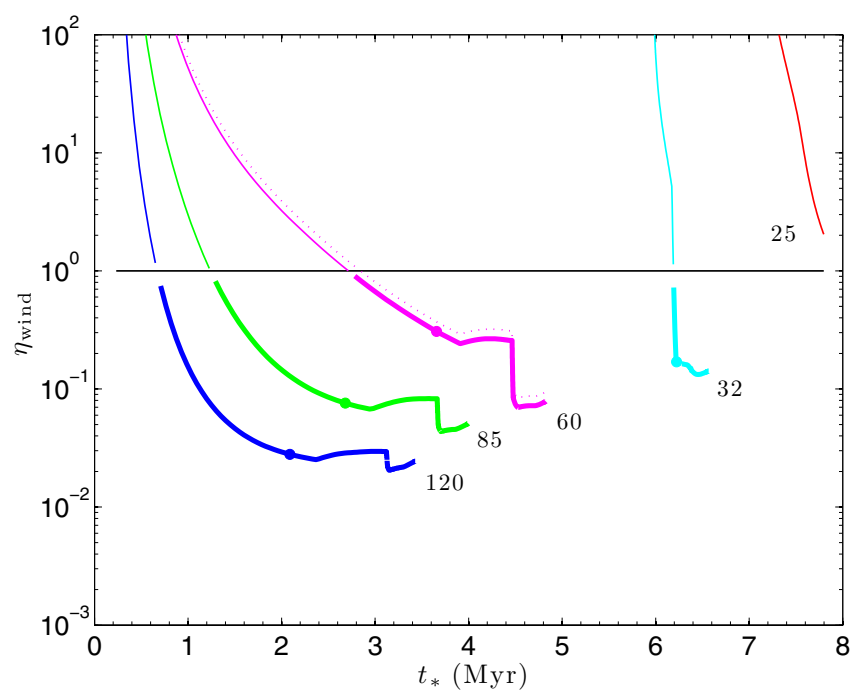

Fig. 4. Thick lines show the acceptable model solutions, i.e. the values of $t_{\star}$ and $\eta_{\text {wind }}$ for which the third generation stars have a solar ${ }^{26} \mathrm{Al}$ abundance. By definition $\eta_{\text {wind }}$ cannot be larger than 1 . The filled circle shows for information the onset of the WR phase. The dotted line shows the $60 M_{\odot}$ model with $\Delta_{C}=0.5 \mathrm{Myr}$ instead of $0.3 \mathrm{Myr}$ (see text).

the collect and collapse phases, i.e. at a time $t_{\star}+\Delta_{C}$ (Fig. 2), we have

$C_{\odot}\left[{ }^{26} \mathrm{Al}\right]=\frac{\eta_{\text {wind }} P_{\text {wind }}\left[{ }^{26} \mathrm{Al}\right]\left(t_{\star}\right)}{M_{\text {shell }}} \mathrm{e}^{-\Delta_{C} / \tau_{26}}$,

where $C_{\odot}\left[{ }^{26} \mathrm{Al}\right]$ is the ${ }^{26} \mathrm{Al}$ abundance at the onset of solar system formation. The last term of the equation accounts for the decay of ${ }^{26} \mathrm{Al}$ during a time $\Delta_{C}$, which is the duration of the final collapse phase, when the ${ }^{26} \mathrm{Al}$-carrying wind is conservatively assumed not to penetrate the shell because it has become too dense (Fig. 2). With $M_{\text {shell }}=1000 M_{\odot}$ (Zavagno et al. 2007), $\Delta_{C}=0.3 \mathrm{Myr}$ (Ward-Thompson et al. 2007) and $C_{\odot}\left[{ }^{26} \mathrm{Al}\right]=3.3 \mathrm{ppb}$ (see Sect. 1), Eq. (2) can be solved for $\eta_{\text {wind }}$ and $t_{\star}$ (Fig. 4). 
As long as $M \geq 32 M_{\odot}$, there is a wide diversity of solutions to our problem (Fig. 4). An acceptable solution (among many others) is for example enrichment by a $85 M_{\odot}$ star during $t_{\star}=3 \mathrm{Myr}$ with an injection efficiency $\eta_{\text {wind }}=0.07$. The acceptable duration of enrichment $\left(t_{\star}\right.$, see Fig. 2) lasts from 0.65 to $6.2 \mathrm{Myr}$, while $\eta_{\text {wind }}$ ranges from $2.1 \times 10^{-2}$ to 1 . For many solutions, ${ }^{26} \mathrm{Al}$-enrichment ends before the onset of the WR phase; i.e., there are many values of $t_{\star}$ that are lower than $t_{\mathrm{WR}}$, where $t_{\mathrm{WR}}$ is the time of entry in the WR phase (see Fig. 4).

\subsection{Constraining generation \#2: the parent cluster size}

Unlike other models, we can constrain the size of the parent cluster of our solar system. If cluster \#2 contains many massive stars, it is likely that a super wind bubble (Parizot et al. 2004) will form and that it will open into the ISM (Güdel et al. 2008), provoking leakage of ${ }^{26} \mathrm{Al}$ atoms. Nice spherical shells are actually observed around single massive stars (Deharveng et al. 2010) rather than around large clusters (Güdel et al. 2008). We can therefore constrain the size $\left(N_{2}\right)$ of the cluster \#2 in imposing two requirements: 1) cluster \#2 contains fewer than five stars more massive than $8 M_{\odot}$ because superbubbles form when the number of massive stars is larger than $n_{\mathrm{SB}}=5$ (Higdon \& Lingenfelter 2005). 2) Cluster \#2 contains at least one star more massive than $32 M_{\odot}$, the minimum mass for which Eq. (2) has a solution (Fig. 4). We call $\mathcal{P}(\mathrm{N})$ the size-dependent probability distribution of a cluster containing $N$ stars and satisfying conditions 1 ) and 2).

To calculate $\mathcal{P}(\mathrm{N})$ we generate, for a given cluster size, 10000 realizations of the stellar initial mass function (IMF), and count the fraction of realizations which satisfy the two constraints exposed above. We use the IMF of Kroupa et al. (1993) mimicked by the generating function:

$M=0.08+\left(0.19 \xi^{1.55}+0.05 \xi^{0.6}\right) /\left(1-\xi^{0.58}\right)$,

where $\xi$ is a random number to be chosen between 0 and 1 (Kroupa et al. 1993). We consider only those distributions for which the most massive star is less massive than $150 M_{\odot}$, a likely upper limit for stellar masses (Weidner \& Kroupa 2006). Using that generating function, the fraction $f_{\mathrm{SN}}$ of stars more massive than $8 M_{\odot}$ (i.e. which will go SN) is $2.4 \times 10^{-3}$, and the average stellar mass is $M_{\star}=0.5 M_{\odot}$.

The calculated distribution probability is shown in Fig. 5. It is bell-shaped, and clusters with size $\sim 1200$ stars have the highest probability $(\sim 15 \%)$ to satisfy the dual constraint evoked above. In $10 \%$ of the positive runs, clusters with 1200 stars contain two stars more massive than $32 M_{\odot}$, which would both contribute to the ${ }^{26} \mathrm{Al}$ enrichment. In such occurrences, the increased amount of ${ }^{26} \mathrm{Al}$ would relax the (already loose) constraint on $\eta_{\text {wind }}$.

\section{Origin of ${ }^{60} \mathrm{Fe}$ in the solar system}

\subsection{Updating the SPACE model}

In our generalized model, ${ }^{60} \mathrm{Fe}$ originates in $\mathrm{SNe}$ from the first generation of stars (see panel $a$ in Fig. 1), as proposed in the supernova propagation and cloud enrichment (SPACE) model (Gounelle et al. 2009). The calculations presented here are modified when taking the new (longer) mean lifetime of ${ }^{60} \mathrm{Fe}$ (Rugel et al. 2009) into account, and adopting the SN yields of Woosley \& Heger (2007) rather than a combination of yields as in Gounelle et al. (2009).

We consider a set of $N_{1}$ stars coevally formed at time 0 and distributed in mass according to the initial mass function.

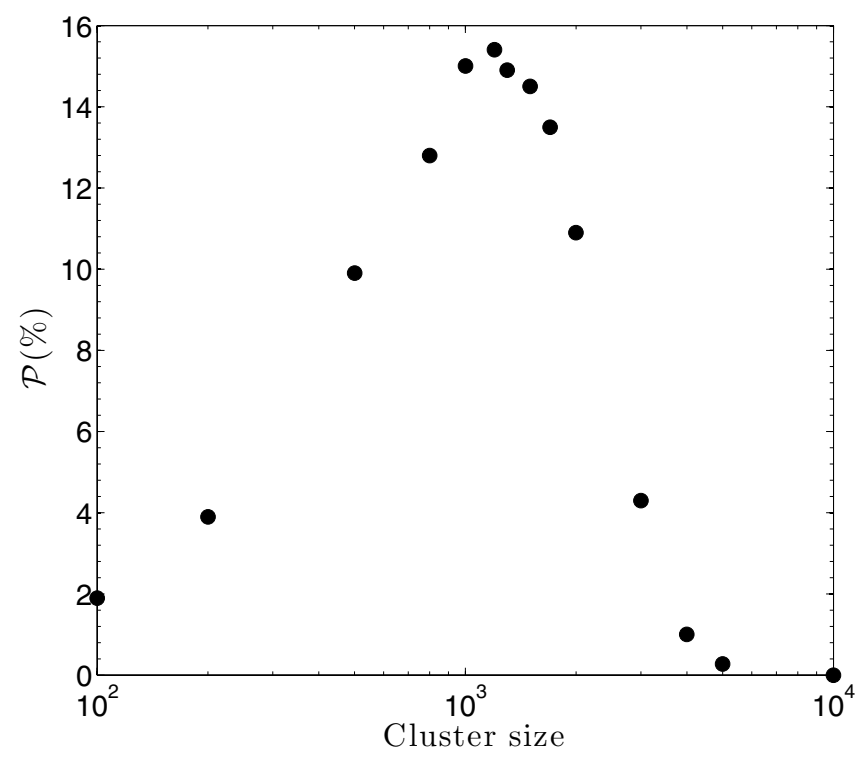

Fig. 5. Probability $\mathcal{P}$ (in $\%$ ), as a function of the cluster size $\mathrm{N}$, to realize the double condition: 1$)$ the number of massive stars $\left(M \geq 8 M_{\odot}\right)$ is below $n_{\mathrm{SB}}=5$ and 2 ) one star at least has a mass over $32 M_{\odot}$. For each point, the IMF (see text) was simulated 10000 times.

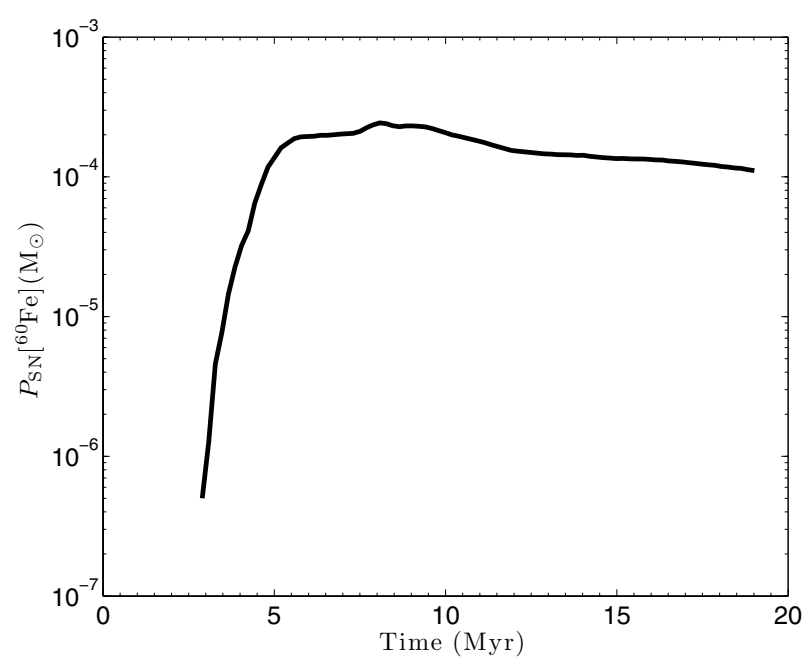

Fig. 6. $P_{\mathrm{SN}}\left[{ }^{60} \mathrm{Fe}\right]\left(N_{1}, \mathrm{t}\right)$ for $N_{1}=5000$ stars, averaged over 1000 realizations of the IMF. The slight difference with Fig. 2 of Gounelle et al. (2009) is due to the longer mean lifetime of ${ }^{60} \mathrm{Fe}$.

The time-dependent amount of ${ }^{60} \mathrm{Fe}$ ejected by SNe in the ISM, $P_{\mathrm{SN}}\left[{ }^{60} \mathrm{Fe}\right]\left(N_{1}, t\right)$, writes as

$P_{\mathrm{SN}}\left[{ }^{60} \mathrm{Fe}\right]\left(N_{1}, t\right)=\sum_{i=1}^{N_{\mathrm{SN}}} Y_{\mathrm{SN}_{\mathrm{i}}}\left({ }^{60} \mathrm{Fe}\right) \mathrm{e}^{-\frac{\left(t-t_{i}\right)}{\tau_{60}}}$,

where $Y_{\mathrm{SN}_{\mathrm{i}}}\left({ }^{60} \mathrm{Fe}\right)$ are the ${ }^{60} \mathrm{Fe}$ yields of the $i$ th $\mathrm{SN}, t_{i}$ is the explosion time of the $i$ th $\mathrm{SN}, \tau_{60}$ is the ${ }^{60} \mathrm{Fe}$ mean lifetime, and $N_{\mathrm{SN}}$ is the total number of $\mathrm{SNe}$ (i.e. stars more massive than $8 M_{\odot}$ ) from generation \#1. The massive stars lifetimes are those calculated by Schaller et al. (1992).

To fix ideas, we adopt $N_{1}=5000$ stars and perform the calculation 1000 times to take the stochastic nature of star formation into account. The evolution of $P_{\mathrm{SN}}\left[{ }^{60} \mathrm{Fe}\right](5000)$ with time is shown in Fig. 6. It takes roughly 5 Myr to establish a steady state abundance of ${ }^{60} \mathrm{Fe}$ (due to the balance of SN production and decay). We define the background (steady-state) 
value as $\hat{P}_{\mathrm{SN}}\left[{ }^{60} \mathrm{Fe}\right]\left(N_{1}\right)=P_{\mathrm{SN}}\left[{ }^{60} \mathrm{Fe}\right]\left(N_{1}, 10 \mathrm{Myr}\right)$, i.e. at the onset of star formation of generation \#2. In our fiducial case, $\hat{P}_{\mathrm{SN}}\left[{ }^{60} \mathrm{Fe}\right](5000)=2.1 \times 10^{-4} M_{\odot}$ (Fig. 6). For each cluster size, 10000 realizations of the IMF were made to check the dependency of $\hat{P}_{\mathrm{SN}}\left[{ }^{60} \mathrm{Fe}\right]$ with $N_{1}$. It is obviously linear and we find $\hat{P}_{\mathrm{SN}}\left[{ }^{60} \mathrm{Fe}\right]\left(N_{1}\right)=p_{\mathrm{SN}}\left[{ }^{60} \mathrm{Fe}\right] \times N_{1}$, with $p_{\mathrm{SN}}\left[{ }^{60} \mathrm{Fe}\right]=$ $4.2 \times 10^{-8} M_{\odot}$.

A GMC region having a mass $M_{2}$ receiving $\hat{P}_{\mathrm{SN}}\left[{ }^{60} \mathrm{Fe}\right]$ solar masses of ${ }^{60} \mathrm{Fe}$ will have an ${ }^{60} \mathrm{Fe}$ concentration of $\mathrm{C}\left[{ }^{60} \mathrm{Fe}\right]=\gamma_{\mathrm{SN}}$ $\hat{P}_{\mathrm{SN}}\left[{ }^{60} \mathrm{Fe}\right] / M_{2}=\gamma_{\mathrm{SN}} \times p_{\mathrm{SN}}\left[{ }^{60} \mathrm{Fe}\right] N_{1} / M_{2}$, where $\gamma_{\mathrm{SN}}$ is the dilution factor of the ejecta in region \#2 and $N_{2}$ the number of stars formed in region \#2. With $M_{2}=M_{\star} N_{2} / \epsilon$ where $\epsilon$ is the star formation efficiency and $\mathrm{M}_{\star}$ is the average mass of a star (see Sect. 3.3), one obtains the simple relationship

$C\left[{ }^{60} \mathrm{Fe}\right]=\gamma_{\mathrm{SN}} p_{\mathrm{SN}}\left[{ }^{60} \mathrm{Fe}\right] N_{1} \frac{\epsilon}{N_{2} M_{\star}}$

With $p_{\mathrm{SN}}\left[{ }^{60} \mathrm{Fe}\right]=4.2 \times 10^{-8} M_{\odot}, \epsilon=30 \%$ (Elmegreen 2007 ; Lada \& Lada 2003) and $M_{\star}=0.5 M_{\odot}$ (see Sect. 3.3), it gives the relationship

$C\left[{ }^{60} \mathrm{Fe}\right]=25.210^{-9} \gamma_{\mathrm{SN}} \frac{N_{1}}{N_{2}} M_{\odot} / M_{\odot}$.

Imposing that, at the onset of star formation in generation \#3, the ${ }^{60} \mathrm{Fe}$ concentration established by the $N_{1}$ stars from the first generation of stars is equal to that of the nascent solar system $\left(C_{\odot}\left[{ }^{60} \mathrm{Fe}\right]\right)$, Eq. (6) reads as

$C_{\odot}\left[{ }^{60} \mathrm{Fe}\right]=25.2 \gamma_{\mathrm{SN}} \frac{N_{1}}{N_{2}} \mathrm{e}^{-t / \tau_{60}} \mathrm{ppb}$,

where $t$ is the time elapsed between the onset of star generation \#2 and of generation \#3 (that of our Sun).

\subsection{Constraining generation \#1: the grandparent cluster size}

The quantity $t$ in Eq. (7), which is the duration between the onset of star generation \#2 and of generation \#3 can be written as the sum of $t_{\star}$ and of $\Delta_{C}$. With $N_{2}=1200$ stars (see Sect. 3.3) and $0.65 \leq t_{\star} \leq 6.2 \mathrm{Myr}$ (see Sect. 3.2), we obtain $26 \leq \gamma_{\mathrm{SN}} N_{1} \leq 108$ or, to account for a possible revision of the initial solar system content of ${ }^{60} \mathrm{Fe}$, expressed as a function of the initial ratio $\left({ }^{60} \mathrm{Fe} /{ }^{56} \mathrm{Fe}\right)_{0}$ :

$26 \frac{\left({ }^{60} \mathrm{Fe} /{ }^{56} \mathrm{Fe}\right)_{0}}{3 \times 10^{-7}} \leq \gamma_{\mathrm{SN}} N_{1} \leq 108 \frac{\left({ }^{60} \mathrm{Fe} /{ }^{56} \mathrm{Fe}\right)_{0}}{3 \times 10^{-7}}$.

The range of possible solutions $\left(N_{1}, \gamma_{\mathrm{SN}}\right)$ is shown in Fig. 7. A first generation cluster with $N_{1}=5000$ stars and $\gamma_{\mathrm{SN}}=0.02$ is a possible reasonable solution. If, as recently suggested (Moynier et al. 2011), the solar system initial abundance of ${ }^{60} \mathrm{Fe}$ was lower than the one usually accepted, $\gamma_{\mathrm{SN}}$ or $N_{1}$ could accordingly be decreased.

\section{The origin of SLRs: a generalized model}

It is therefore possible to identify the sequence of events that account for the presence of SLRs with a variety of mean lifetimes in the nascent solar system (Fig. 1).

- SLRs with the longest mean lifetimes ( $25 \mathrm{Myr}$ ) and with abundance compatible with a Galactic background origin, come from a large diversity of stars on the (kpc) scale of star complexes on a few $10 \mathrm{~s}$ of Myr timescales (Huss et al. 2009).

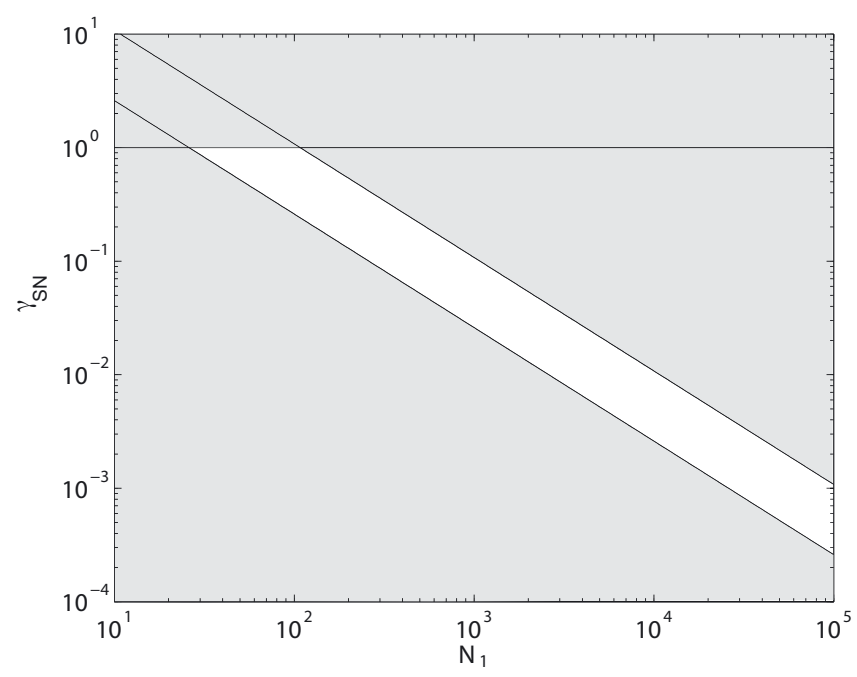

Fig. 7. The dilution factor, $\gamma_{\mathrm{SN}}$ vs. $N_{1}$, the number of stars in star generation \#1. The allowed range of parameters is depicted in white and defined by $\gamma_{\mathrm{SN}} \leq 1$ (by construction) and $26 \leq \gamma_{\mathrm{SN}} N_{1} \leq 108$ (see Sect. 4.2).

- ${ }^{60} \mathrm{Fe}$ in the solar system was synthesized by a handful of SNe on the GMC (10 s of pc) scale on a 5-10 Myr timescale.

- ${ }^{26} \mathrm{Al}$ was finally delivered by the wind of a single massive $\left(M \gtrsim 32 M_{\odot}\right)$ star on a few Myr timescale and on a 5-10 pc spatial scale.

The variability of temporal scales recorded by SLRs' mean lifetimes corresponds to the diversity of spatial scales at which star formation is observed (Elmegreen 2007): the longer the mean lifetime, the larger the scale of injection in the ISM. This spatio/temporal variability is simply the reflection of sequential star formation in a hierarchical ISM (Elmegreen 2007). Sequential star formation is an important mechanism of star formation that has been known for a long time (Elmegreen \& Lada 1977; Elmegreen 2007; Hennebelle et al. 2009). It is observed in a fossilized form in star-forming regions such as Orion (Bally 2008) or Scorpius-Centaurus (Preibisch \& Zinnecker 2007), where OB subgroups with different ages are interpreted to have arisen from propagating star formation. It has also been caught in the act at the borders of HII regions (Deharveng et al. 2010; Snider et al. 2009), as well as on larger scales in the Carina star-forming region (Chen et al. 2007).

Though occurring on a wide range of spatial scales, a similar mechanism accounts for the origins of ${ }^{26} \mathrm{Al}$ and ${ }^{60} \mathrm{Fe}$. For both radionuclides, ISM gas is collected by the same agent as the one that carries the SLR (SNe ejecta or massive star wind). After it has become dense enough, the collected gas is unstable to gravity and collapses to eventually form new stars. The SLRs that were contained in the collecting agent end up in the new generation of stars. In other words, given that the SNe shocks and massive star winds that contribute to the building of dense phases of the ISM also carry radioactive elements, it is not surprising that molecular clouds - and stars formed within them are radioactive.

\section{Discussion}

Our model therefore provides a natural explanation for the elusive presence of ${ }^{26} \mathrm{Al}$ and ${ }^{60} \mathrm{Fe}$ in the nascent solar system. It is generic because, both for ${ }^{26} \mathrm{Al}$ and ${ }^{60} \mathrm{Fe}$, a collectand-collapse mechanism (Elmegreen \& Lada 1977) is at work, 
though operating on different scales. It does not suffer from the other difficulties encountered by the previous models. Because the ${ }^{60} \mathrm{Fe}$ and ${ }^{26} \mathrm{Al}$ have a decoupled origin (SN and wind, respectively), our model is not faced with the problem of the low ${ }^{26} \mathrm{Al} /{ }^{60} \mathrm{Fe}$ ratio met by the single $\mathrm{SN}$ models (Ouellette et al. 2005; Boss et al. 2010). In addition, when the massive star goes $\mathrm{SN}$, the disk is far enough away $(5-10 \mathrm{pc})$ to avoid the disruption caused by the SN shockwave (Chevalier 2000) or overinjection of ${ }^{60} \mathrm{Fe}$, unlike the model of Gaidos et al. (2009). More importantly, it agrees with the astronomical observations of starforming regions and the accepted astrophysical mechanisms of star formation.

Our model leaves room for some imperfect mixing to account for the injection of ${ }^{26} \mathrm{Al}$ by a stellar wind ( $\left.\eta_{\text {wind }} \leq 1\right)$, unlike previous models that assume perfect mixing (Gaidos et al. 2009; Tatischeff et al. 2010). Though it would be desirable to perform numerical simulations to calculate dust and gas injection efficiencies from the first principles, the values required in our model (as low as 0.02) can be reached easily. In fact, it was shown that the injection efficiency, at least for $\mathrm{SNe}$ ejecta, was multiplied by a factor of 3 when density was decreased by a factor of 2 (Boss et al. 2010). By construction, the collected shell has a lower density than that of a collapsing core, for which injection efficiencies are as high as 0.02 (Boss \& Keiser 2010).

The model is quite robust to parameter changes. First of all, it does not pretend to give a unique solution but rather a range of possible solutions. This diversity of solutions is intellectually satisfying since it would be fairly vain to pretend to exactly identify the star(s) responsible for the solar system ${ }^{26} \mathrm{Al}$ and ${ }^{60} \mathrm{Fe}$ enrichment relative to the Galactic background. It also means that there is ample room for parameter changes. Second, we have been quite conservative in the choice of our model inputs. This is exemplified by the adoption of new stellar models with metallicity lower than the one adopted by previous works. This lower metallicity implies lower initial content of ${ }^{25} \mathrm{Mg}$, which is the nucleus whose transformation produces ${ }^{26} \mathrm{Al}$ during the core $\mathrm{H}$-burning phase, and thus our models produce smaller quantities of ${ }^{26} \mathrm{Al}$ than the models of Limongi \& Chieffi (2006) and Palacios et al. (2005) used in previous work (Arnould et al. 1997; Gaidos et al. 2009; Tatischeff et al. 2010). On the same line, we have conservatively considered that, during the final phase of collapse lasting $\Delta_{C}=0.3 \mathrm{Myr}$, the shell was closed to ${ }^{26} \mathrm{Al}$ injection, unlike, for example, the models of Boss et al. (2010). Adopting an even longer isolation time would not change our conclusions (see the model with $\Delta_{C}=0.5 \mathrm{Myr}$ in Fig. 4). The range of values found for $t_{\star}$ is compatible with the observed timescales for the onset of star formation in a collected shell (Deharveng et al. 2010). The shell mass agrees with observations (Zavagno et al. 2007; Deharveng et al. 2008) though it could be a factor of two higher (Zavagno et al. 2007; Deharveng et al. 2008). In any case, the assumed mass of the shell is $10^{5}$ times higher than the disk of Ouellette et al. (2005), 500 times more than the core of Boss \& Keiser (2010), and four times greater than the most massive bow-shock shell of Tatischeff et al. (2010). It means that the probability of enrichment (not even taking the astrophysical context into account, Gounelle \& Meibom 2008), for a given massive star, is far higher in our collect-injectionand-collapse model than in any other model, considering that a single massive star is responsible for the solar system's ${ }^{26} \mathrm{Al}$.

On a slightly different note, our reasoning does not critically depend on adopting the Woosley \& Heger (2007) SN yields. These were chosen because they are based on the solar abundances of Lodders (2003), which are almost identical to the newly determined solar abundances of Asplund et al. (2009),

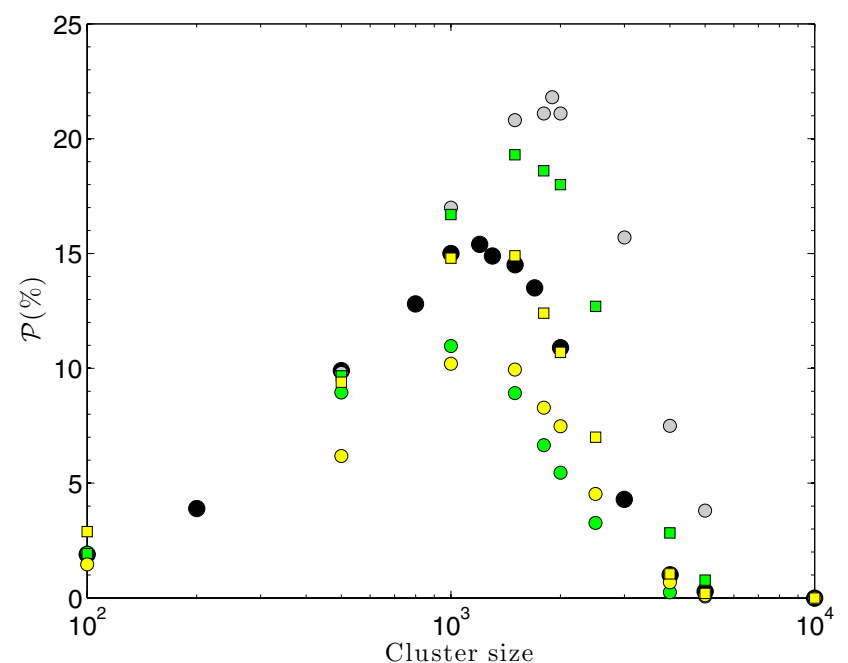

Fig. 8. Probability $\mathcal{P}$ (in \%), as a function of the cluster size $N$, to realize the double condition: 1) the number of stars with mass higher than $M_{\mathrm{SN}}$ is smaller than $n_{\mathrm{SB}}$ and 2) one star at least has a mass greater than $M_{\min }$, where the values $n_{\mathrm{SB}}, M_{\min }$, and $M_{\mathrm{SN}}$ are set to vary. For each point, the IMF (see text) was simulated 10000 times. Filled circles: same as Fig. 5, i.e. $n_{\mathrm{SB}}=5, M_{\min }=32 M_{\odot}$ and $M_{\mathrm{SN}}=8 M_{\odot}$. Gray circles: all being the same but $M_{\mathrm{SN}}=10 M_{\odot}$. Green circles: all being the same but $n_{\mathrm{SB}}=4$. Green squares: all the same but $n_{\mathrm{SB}}=6$. Yellow circles: all the same but $M_{\min }=40 M_{\odot}$. Yellow squares: all the same but $M_{\min }=25 M_{\odot}$.

unlike the older ones of Anders \& Grevesse (1989) used by other modelers of SN yields, such as Limongi \& Chieffi (2006). These new abundances, calculated using 3D hydrodynamical models of the solar atmosphere, are supported by a high degree of internal consistency and by agreement with values obtained in the solar neighborhood, though some discrepancy with heliosismology data persists (Asplund et al. 2009). It is also important to note that, though uncertain, the Woosley \& Heger (2007) SN yields are probably correct within a factor of a few since the IMF integrated ${ }^{26} \mathrm{Al} /{ }^{60} \mathrm{Fe}$ ratio of $\mathrm{SNe}$ is comparable (within a factor of a few) to the one observed in the ISM by the INTEGRAL satellite (Wang et al. 2007). Finally, adopting different yields (such as those of Limongi \& Chieffi 2006) would change the results by a factor of a few, not critical in the reasonings of Sects. 1 or 4 , especially given the debates concerning the initial abundance of ${ }^{60} \mathrm{Fe}$ (see below).

An important aspect of our model is that it constrains the solar system genealogy, i.e., it provides estimates of the number of stars contained in the parent and grandparent clusters $\left(N_{2}\right.$ and $N_{1}$, respectively). These cluster sizes are not constrained in the same fashion. In either of these cases, it is important to keep in mind that we only have access to an order of magnitude, as expected for a stellar nursery that was active $4.5 \mathrm{Gyr}$ ago and whose only memory is preserved in meteorites' most primitive phases.

The best estimate of $N_{2}$ depends on a variety of numerical values as described in Sect. 3.3, namely the minimum number of stars, $n_{\mathrm{SB}}$, needed to initiate a super wind bubble expansion (identified to the minimum number of stars needed to create an SN-blown superbubble since the energy contained in stellar winds is comparable to the energy contained in SNe explosions, Parizot et al. 2004) and the minimum mass for which Eq. (2) has a solution. It also depends on the minimum mass for which a star goes SN which is $M_{\text {min }}=8 M_{\odot}$ but is somehow model dependent (Smartt 2009). To check that dependency, we explored the variations in $\mathcal{P}(\mathrm{N})$ with these parameters (Fig. 8). Even allowing a 
relatively large and unlikely exploration of the parameter space, it is easy to see that the most probable value of $N_{2}$ always lies between 1000 and 2000. This exploration of parameters also encompasses IMF formulations other than the one in Kroupa et al. (1993).

Given the dependency of the size of generation \#1 on the initial abundance of ${ }^{60} \mathrm{Fe}$ and on $\gamma_{\mathrm{SN}}$ (see Eq. (8)), it is obvious that the value of $N_{1}$ is at present poorly known. It is not surprising that the size of the grandparent generation is less precisely constrained than the parent generation. As in any family, the further back in time one goes, the more difficult it is to retrieve precise information on ancestors. More knowledge will be gained on $N_{1}$ when analysts have converged on the initial solar system value of ${ }^{60} \mathrm{Fe}$ (Quitté et al. 2010; Moynier et al. 2011), along with the development of numerical simulations that will help to better constrain the value of $\gamma_{\mathrm{SN}}$.

The model can naturally account for some heterogeneity in the initial distribution of ${ }^{26} \mathrm{Al}$. Injection takes place on only one side of the dense shell. Because some time will be needed for ${ }^{26} \mathrm{Al}$ carriers (gas or solid) to diffuse within the shell entire volume, it is expected that the first solids to form close to the protostar, as expected for CAIs (Krot et al. 2009), will not contain ${ }^{26} \mathrm{Al}$, as is observed in some meteoritic samples (Liu et al. 2009; Makide et al. 2011; Weber et al. 1995). Though the exact diffusion timescale is difficult to quantify without entering desired complex numerical simulations, it is expected to be close to the crossing time of the core, i.e. within the range of 0.5-1 Myr (Bergin \& Tafalla 2007), marginally compatible with the collapse timescale of a few 0.1 Myr (Ward-Thompson et al. 2007). Homogenization mechanisms will take place during the disk phase following the mechanisms presented by Boss (2011). Whether that homogenization goes to completion is at present unknown (Villeneuve et al. 2009; Liu et al. 2012; Gounelle \& Russell 2005). Given that ${ }^{60} \mathrm{Fe}$ production halts some Myr and one star generation before the Sun's formation, its distribution is expected to be homogeneous. At present, there is no experimental consensus on the homogeneous vs. heterogeneous distribution of ${ }^{60} \mathrm{Fe}$ in the early solar system (Dauphas et al. 2008; Quitté et al. 2010).

Our model produces no undesired collateral enrichments (Nichols et al. 1999; Gounelle \& Meibom 2007). Besides ${ }^{26} \mathrm{Al}$, massive stars winds do not contain other SLRs until the very last $3 \times 10^{5} \mathrm{yr}$ of the star's life (Arnould et al. 2006), during which ${ }^{26} \mathrm{Al}$ injection is not considered. Given the high dilution factors of the wind needed to account for ${ }^{26} \mathrm{Al}\left(\leq 10^{-3}\right)$, the oxygen isotopic composition of the shell is not modified. The shift in the oxygen isotopic composition is calculated to be less than a few per mil for all the considered models, which is far below our detection capability.

\section{Conclusion and implications}

We have elucidated the origin of SLRs in the early solar system by developing a new model for ${ }^{26} \mathrm{Al}$ that relies on a physical mechanism (collect + injection, collapse) similar in essence to the one presented recently for ${ }^{60} \mathrm{Fe}$ (Gounelle et al. 2009). This new mechanism occurs naturally within a common mode of star formation, namely that of sequential star formation within a giant molecular cloud (Hennebelle et al. 2009). Within a few milligrams of meteorites, SLRs therefore record physical mechanisms observed in the sky on scales varying from hundreds down to $1 \mathrm{pc}$.

The identified sequence of events establishes the genealogy of the solar system. Our Sun is the great-grandson of a star complex (generation 0) containing 10s of thousands of stars, the grandson of a large GMC core (generation 1) containing a few thousand stars, and the son of a massive $\left(\gtrsim 32 M_{\odot}\right)$ star belonging to a cluster (1000-2000 stars with a preferred value of 1200 stars, generation \#2) born later within the same GMC. Assuming 30\% star formation efficiency (Lada \& Lada 2003) and with an average stellar mass of $M_{\star}=0.5 M_{\odot}$, our Sun was born together with $\sim$ six hundred fellow stars in its natal $1000 M_{\odot}$ shell. Formation of the Sun in a relatively small cluster is in agreement with dynamical requirements, i.e. stability of planetary orbits, existence of the Kuiper belt object Sedna, and formation of the Oort cloud (Adams 2010; Brasser et al. 2012).

Given the size of its cluster at birth (six hundred stars), it is not inconceivable that our Sun was coeval to a massive star. Since the average number of massive stars in a cluster of size $N$ is $f_{\mathrm{SN}} \times N$, with $f_{\mathrm{SN}}=2.3 \times 10^{-3}$, clusters with six hundred stars might contain between 1 or 2 massive stars, most probably B stars, leaving room for disk photo evaporation as suggested by Throop \& Bally (2005).

It is expected that the few hundred stars born together with our Sun will share the same chemical and isotopic properties. These stars were true twins of our Sun. Given that stellar clusters dissipate on timescales of $100 \mathrm{Myr}$ (Adams et al. 2001), that revolution timescales around the Galactic center are on the order of $200 \mathrm{Myr}$, and that stars can radially migrate over a few $\mathrm{kpc}$ in the Galaxy (Roškar et al. 2008), these fellow stars are now in totally unrelated places. Because the ${ }^{26} \mathrm{Al}$ enrichment mechanism we have identified is generic, many other stars are, however, expected to contain ${ }^{26} \mathrm{Al}$ at a level close to that of our solar system.

Acknowledgements. We sincerely thank an anonymous reviewer for very valuable comments that significantly helped to improve the paper. We are grateful to Thierry Montmerle for fruitful discussions, to Sébastien Fromang for insights on dense core timescales as well as to Marc Chaussidon, Alessandro Morbidelli, and Edward Young for critical readings of earlier versions of the manuscript. Figures were kindly produced by Michel Serrano. This work is partly supported by the Programme National de Planétologie (PNP).

\section{References}

Adams, F. C. 2010, ARA\&A, 48, 47

Adams, J. D., Stauffer, J. R., Monet, D. G., Skrutskie, M. F., \& Beichman, C. A. 2001, AJ, 121, 2053

Anders, E., \& Grevesse, N. 1989, Geochim. Cosmochim. Acta, 53, 197

Arnould, M., Paulus, G., \& Meynet, G. 1997, A\&A, 321, 452

Arnould, M., Goriely, S., \& Meynet, G. 2006, A\&A, 453, 653

Asplund, M., Grevesse, N., Sauval, A. J., \& Scott, P. 2009, ARA\&A, 47, 481

Bally, J. 2008, Overview of the Orion Complex, ed. B. Reipurth, 459

Bergin, E. A., \& Tafalla, M. 2007, ARA\&A, 45, 339

Birck, J. L., \& Lugmair, G. W. 1988, Earth Planet. Sci. Lett., 90, 131

Boss, A. P. 2011, ApJ, 739, 61

Boss, A. P., \& Keiser, S. A. 2010, ApJ, 717, L1

Boss, A. P., Ipatov, S. I., Keiser, S. A., Myhill, E. A., \& Vanhala, H. A. T. 2008, ApJ, 686, L119

Boss, A. P., Keiser, S. A., Ipatov, S. I., Myhill, E. A., \& Vanhala, H. A. T. 2010, ApJ, 708, 1268

Brasser, R., Duncan, M. J., Levison, H. F., Schwamb, M. E., \& Brown, M. E. 2012 , Icarus, 217,1

Bricker, G. E., \& Caffee, M. W. 2010, ApJ, 725, 443

Cameron, A. G. W., \& Truran, J. W. 1977, Icarus, 30, 447

Cameron, A. G. W., Hoeflich, P., Myers, P. C., \& Clayton, D. D. 1995, ApJ, 447, L53

Chen, W. P., Lee, H. T., \& Sanchawala, K. 2007, in IAU Symp. 237, eds. B. G. Elmegreen, \& J. Palous, 278

Chevalier, R. A. 2000, ApJ, 538, L151

Crowther, P. A. 2007, ARA\&A, 45, 177

Dauphas, N., Cook, D. L., Sacarabany, A., et al. 2008, ApJ, 686, 560

Deharveng, L., Lefloch, B., Kurtz, S., et al. 2008, A\&A, 482, 585 
Deharveng, L., Zavagno, A., Schuller, F., et al. 2009, A\&A, 496, 177 Deharveng, L., Schuller, F., Anderson, L. D., et al. 2010, A\&A, 523, A6 de Jager, C., Nieuwenhuijzen, H., \& van der Hucht, K. A. 1988, A\&AS, 72, 259 Desch, S. J., Connolly, H. C., Jr., \& Srinivasan, G. 2004, ApJ, 602, 528 Desch, S. J., Pan, L., \& Scannapieco, E. 2011, LPI Contributions, 1639, 9117 Duprat, J., \& Tatischeff, V. 2007, ApJ, 671, L69

Elmegreen, B. G. 2007, ApJ, 668, 1064

Elmegreen, B. G., \& Lada, C. J. 1977, ApJ, 214, 725

Gaidos, E., Krot, A. N., Williams, J. P., \& Raymond, S. N. 2009, ApJ, 696, 1854

Gounelle, M., \& Meibom, A. 2007, ApJ, 664, L123

Gounelle, M., \& Meibom, A. 2008, ApJ, 680, 781

Gounelle, M., \& Meibom, A. 2010, in EAS Pub. Ser., 41, eds. T. Montmerle, D. Ehrenreich, \& A.-M. Lagrange, 301

Gounelle, M., \& Russell, S. S. 2005, Geochim. Cosmochim. Acta, 69, 3129

Gounelle, M., Shu, F. H., Shang, H., et al. 2006, ApJ, 640, 1163

Gounelle, M., Meibom, A., Hennebelle, P., \& Inutsuka, S.-i. 2009, ApJ, 694, L1

Güdel, M., Briggs, K. R., Montmerle, T., et al. 2008, Science, 319, 309

Hartmann, L. 2005, in Chondrites and the Protoplanetary Disk, eds. A. N. Krot, E. R. D. Scott, \& B. Reipurth, ASP Conf. Ser., 341, 131

Hayashi, C., Nakazawa, K., \& Nakagawa, Y. 1985, Protostars and planets II (Tucson, AZ: University of Arizona Press), 1100

Hennebelle, P., Mac Low, M.-M., \& Vazquez-Semadeni, E. 2009, Diffuse interstellar medium and the formation of molecular clouds, ed. G. Chabrier (Cambridge University Press), 205

Higdon, J. C., \& Lingenfelter, R. E. 2005, ApJ, 628, 738

Huss, G. R., Meyer, B. S., Srinivasan, G., Goswami, J. N., \& Sahijpal, S. 2009, Geochim. Cosmochim. Acta, 73, 4922

Jacobsen, B., Yin, Q.-Z., Moynier, F., et al. 2008, Earth Planet. Sci. Lett., 272, 353

Jacobsen, B., Matzel, J., Hutcheon, I. D., et al. 2011, ApJ, 731, L28

Kastner, J. H., \& Myers, P. C. 1994, ApJ, 421, 605

Koyama, H., \& Inutsuka, S.-i. 2002, ApJ, 564, L97

Krot, A. N., Amelin, Y., Bland, P., et al. 2009, Geochim. Cosmochim. Acta, 73, 4963

Kroupa, P., Tout, C. A., \& Gilmore, G. 1993, MNRAS, 262, 545

Lada, C. J., \& Lada, E. A. 2003, ARA\&A, 41, 57

Lee, T., Papanastassiou, D. A., \& Wasserburg, G. J. 1976, Geophys. Res. Lett., 3,41

Limongi, M., \& Chieffi, A. 2006, ApJ, 647, 483

Liu, M.-C., McKeegan, K. D., Goswami, J. N., et al. 2009, Geochim. Cosmochim. Acta, 73, 5051

Liu, M.-C., Chaussidon, M., Göpel, C., \& Lee, T. 2012, Earth Planet. Sci. Lett., 327,75

Lodders, K. 2003, ApJ, 591, 1220

Lugaro, M., et al. 2012, MAPS, in press

MacPherson, G. J., Davis, A. M., \& Zinner, E. K. 1995, Meteoritics, 30, 365

Maeder, A. 2003, A\&A, 399, 263

Maeder, A., \& Meynet, G. 2000, A\&A, 361, 159

Makide, K., Nagashima, K., Krot, A. N., et al. 2011, ApJ, 733, L31

McKeegan, K. D., \& Davis, A. M. 2005, Early Solar System Chronology, eds. A. M. Davis, H. D. Holland, \& K. K. Turekian (Elsevier B), 431

Meyer, B. S., \& Clayton, D. D. 2000, Space Sci. Rev., 92, 133

Meynet, G., \& Maeder, A. 2002, A\&A, 390, 561
Meynet, G., Ekström, S., Maeder, A., et al. 2008, in IAU Symp. 250, eds. F. Bresolin, P. A. Crowther, \& J. Puls, 147

Moynier, F., Blichert-Toft, J., Wang, K., Herzog, G. F., \& Albarede, F. 2011, ApJ, 741, 71

Nichols, R. H., Jr., Podosek, F. A., Meyer, B. S., \& Jennings, C. L. 1999, Meteorit. Planet. Sci., 34, 869

Nugis, T., \& Lamers, H. J. G. L. M. 2000, A\&A, 360, 227

Ouellette, N., Desch, S. J., Hester, J. J., \& Leshin, L. A. 2005, Chondrites and the Protoplanetary Disk, 341, 527

Ouellette, N., Desch, S. J., Bizzarro, M., et al. 2009, Geochim. Cosmochim. Acta, 73, 4946

Palacios, A., Meynet, G., Vuissoz, C., et al. 2005, A\&A, 429, 613

Parizot, E., Marcowith, A., van der Swaluw, E., Bykov, A. M., \& Tatischeff, V. 2004, A\&A, 424, 747

Preibisch, T., \& Zinnecker, H. 2007, in IAU Symp. 237, eds. B. G. Elmegreen, \& J. Palous, 270

Quitté, G., Halliday, A. N., Meyer, B. S., et al. 2007, ApJ, 655, 678

Quitté, G., Markowski, A., Latkoczy, C., Gabriel, A., \& Pack, A. 2010, ApJ, 720, 1215

Roškar, R., Debattista, V. P., Quinn, T. R., Stinson, G. S., \& Wadsley, J. 2008, ApJ, 684, L79

Rugel, G., Faestermann, T., Knie, K., et al. 2009, Phys. Rev. Lett., 103, 072502

Russell, S. S., Gounelle, M., \& Hutchison, R. 2001, Royal Society of London Philosophical Transactions Series A, 359, 1991

Scalo, J. M. 1986, Fund. Cosmic Phys., 11, 1

Schaller, G., Schaerer, D., Meynet, G., \& Maeder, A. 1992, A\&AS, 96, 269

Smartt, S. J. 2009, ARA\&A, 47, 63

Snider, K. D., Hester, J. J., Desch, S. J., Healy, K. R., \& Bally, J. 2009, ApJ, 700,506

Steele, R. C. J., Elliott, T., Coath, C. D., \& Regelous, M. 2011, Geochim. Cosmochim. Acta, 75, 7906

Tachibana, S., \& Huss, G. R. 2003, ApJ, 588, L41

Talon, S., \& Zahn, J.-P. 1997, A\&A, 317, 749

Tatischeff, V., Duprat, J., \& de Séréville, N. 2010, ApJ, 714, L26

Telus, M., Huss, G. R., Nagashima, K., \& Ogliore, R. C. 2011, Meteorit. Planet. Sci. Suppl., 74, 5489

Throop, H. B., \& Bally, J. 2005, ApJ, 623, L149

Trigo-Rodríguez, J. M., García-Hernández, D. A., Lugaro, M., et al. 2009, Meteorit. Planet. Sci., 44, 627

Villeneuve, J., Chaussidon, M., \& Libourel, G. 2009, Science, 325, 985

Vink, J. S., de Koter, A., \& Lamers, H. J. G. L. M. 2000, A\&A, 362, 295

Vink, J. S., de Koter, A., \& Lamers, H. J. G. L. M. 2001, A\&A, 369, 574

Wang, W., Harris, M. J., Diehl, R., et al. 2007, A\&A, 469, 1005

Ward-Thompson, D., André, P., Crutcher, R., et al. 2007, Protostars and Planets V, 33

Wasserburg, G. J., Busso, M., Gallino, R., \& Nollett, K. M. 2006, Nucl. Phys. A, 777,5

Weber, D., Zinner, E., \& Bischoff, A. 1995, Geochim. Cosmochim. Acta, 59, 803

Weidner, C., \& Kroupa, P. 2006, MNRAS, 365, 1333

Williams, J. P., \& Gaidos, E. 2007, ApJ, 663, L33

Woosley, S. E., \& Heger, A. 2007, Phys. Rep., 442, 269

Zavagno, A., Pomarès, M., Deharveng, L., et al. 2007, A\&A, 472, 835 